International Journal of Pure and Applied Mathematics

Volume 95 No. 3 2014, 323-338

ISSN: 1311-8080 (printed version); ISSN: 1314-3395 (on-line version)

url: http://www.ijpam.eu

doi: http://dx.doi.org/10.12732/ijpam.v95i3.1

ijpam.eu

\title{
FUZZY GENERALIZED SEMI GENERALIZED CLOSED SETS
}

\author{
S. Kalaiselvi ${ }^{1 \S}$, V. Seenivasan ${ }^{2}$ \\ ${ }^{1}$ Department of Mathematics \\ University College of Engineering \\ BIT Campus \\ Tiruchirappalli, 620024, Tamilnadu, INDIA \\ ${ }^{2}$ Department of Mathematics \\ University College of Engineering \\ Panruti, 607106, Tamilnadu, INDIA
}

\begin{abstract}
In this paper we introduce a new class of fuzzy set called fuzzy generalized semi generalized closed sets and its characterizations.Besides, we discuss fuzzy gsg-closure and fuzzy gsg-interior with its properties. As an application this set we also introduce fuzzy Tgsg-space. Further, we introduce Fgsg-continuity and Fgsg-irresolute mappings with some of its properties.
\end{abstract}

AMS Subject Classification: 54A40

Key Words: fgsg-closed set, fuzzy gsg-closure, fuzzy Tgsg-space, Fgsgcontiuity.

\section{Introduction}

The usual notation of fuzzy set have been generalized with the introduction of fuzzy sets by Zadeh in his classical paper [22] of 1965. The concepts of fuzzy topological spaces have been introduced and developed by C.L.Chang[5]

Received: December 18, 2013

(C) 2014 Academic Publications, Ltd. url: www.acadpubl.eu

${ }^{\S}$ Correspondence author 
and since then several authors have extended various notions in classical topology to fuzzy topological spaces. The extensions of functions in fuzzy setting can very interestingly and effectively be carried out by the concept of quasicoincidence and q-neighbourhoods introduced by $\mathrm{Pu}$ and Liu [13]. H.Maki et al [9] introduced the concept of generalized closed set in a fuzzy topological space . The concepts Fg $\alpha$-closedsets, Fuzzy generalized semipre closed sets, Fuzzy gs-closed sets, Fuzzy sg-closed sets and Fuzzy semipre generalized closed sets have been investigated in [14-18]. The concept generalized sg-closed sets have been introduced and studied by Lellis et al [8] in classical topology. The purpose of this paper is to extend the notion of generalized sg-closed sets in fuzzy topological spaces. Section 3 is devoted to introduce the fuzzy version of generalized sg-closed sets, generalized sg-open sets and to study some of its properties. In Section 4, we study several interesting characterizations of fuzzy generalized sg-closed sets and fuzzy generalized sg-open sets. We also introduce fuzzy gsg-closure and fuzzy gsg-interior and obtain some of its properties in Section 5. As an application of fuzzy generalized sg-closed set, we introduce fuzzy Tgsg-space in Section 6. In Section 7, we introduce Fgsg-continuous and Fgsg-irresolute mappings by using fuzzy generalized sg-closed set and study some of their fundamental properties.

\section{Preliminaries}

By a fuzzy topological space we shall mean non empty set $\mathrm{X}$ together with fuzzy topology $\tau$ [in the sence of Chang] and denote it by $(\mathrm{X}, \tau)$. Throughout this paper, $(\mathrm{X}, \tau),(\mathrm{Y}, \sigma)$ and $(\mathrm{Z}, \eta)$ ( or simply $\mathrm{X}, \mathrm{Y}$ and $\mathrm{Z}$ ) always mean fuzzy topological spaces.For a fuzzy set $\mathrm{A}$ of $(\mathrm{X}, \tau), \mathrm{Cl}(\mathrm{A}), \operatorname{Int}(\mathrm{A})$ and $1-\mathrm{A}$ denote fuzzy closure, fuzzy interior and fuzzy complement of A respectively. The fuzzy semiclosure ( resp. fuzzy $\alpha$-closure, fuzzy semi-preclosure ) of a fuzzy set A of $(\mathrm{X}, \tau)$ is the intersection of all Fs-closed (resp. F $\alpha$-closed, Fsp-closed) sets that contain $\mathrm{A}$ and is denoted by $\mathrm{sCl}(\mathrm{A})$ (resp. $\alpha \mathrm{Cl}(\mathrm{A})$ and $\mathrm{spCl}(\mathrm{A})$ ).

Definition 2.1. A fuzzy set $A$ of $(X, \tau)$ is called:

1. Fuzzy semiopen (in short, Fs-open) if $A \leq C l(\operatorname{Int}(A))$ and a fuzzy semiclosed (in short, Fs-closed) if $\operatorname{Int}(C l(A)) \leq A[1]$;

2. Fuzzy preopen (in short, $F$ p-open) if $A \leq \operatorname{Int}(\mathrm{Cl}(A))$ and a fuzzy preclosed (in short,Fp-closed) if $C l(\operatorname{Int}(A)) \leq A[4]$;

3. Fuzzy $\alpha$-open (in short, F $\alpha$-open) if $A \leq \operatorname{Int}(\mathrm{Cl}(\operatorname{Int}(A)))$ and a fuzzy $\alpha$-closed (in short, $\mathrm{F} \alpha$-closed) if $\mathrm{Cl}(\operatorname{Int}(\mathrm{Cl}(\mathrm{A}))) \leq A[4]$; 
4. Fuzzy semi-preopen (in short, Fsp-open) if $A \leq C l(\operatorname{Int}(\mathrm{Cl}(\mathrm{A})))$ and a fuzzy semi- preclosed (in short, Fsp-closed) if $\operatorname{Int}(\mathrm{Cl}(\operatorname{Int}(A))) \leq A[22]$.

Lemma 2.2. [11] Let $A$ be a fuzzy set in a fuzzy toplogical space $(X, \tau)$. Then

1. $\operatorname{spCl}(A) \leq \operatorname{sCl}(A) \leq \alpha C l(A) \leq C l(A) \leq r C l(A)$

2. $\operatorname{spCl}(A) \leq p C l(A) \leq \alpha C l(A)$

Definition 2.3. A fuzzy set $A$ of $(X, \tau)$ is called:

1. Fuzzy generalized closed (in short, Fg-closed) [2] if $\mathrm{Cl}(\mathrm{A}) \leq \mathrm{H}$, whenever $A \leq H$ and $H$ is fuzzy open set in $X$;

2. Fuzzy generalized semiclosed (in short, Fsg-closed) [17] if $\mathrm{sCl}_{(A)} \leq H$, whenever $A \leq H$ and $H$ is Fs-open set in $X$. In[6], Hakeim called this set as Generalized fuzzy weakly semiclosed set;

3. Fuzzy generalized semiclosed (in short,Fgs-closed) $[16]$ if $\mathrm{sCl}(\mathrm{A}) \leq \mathrm{H}$, whenever $A \leq H$ and $H$ is fuzzy open set in $X$;

4. Fuzzy $\alpha$-generalized closed (in short,F $\alpha$ g-closed) [12] if $\alpha \mathrm{Cl}(\mathrm{A}) \leq H$, whenever $A \leq H$ and $H$ is $F \alpha$-open set in $X$;

5. Fuzzy generalized $\alpha$-closed (in short,Fg $\alpha$-closed) [12] if $\alpha \mathrm{Cl}(\mathrm{A}) \leq H$, whenever $A \leq H$ and $H$ is fuzzy open set in $X$;

6. Fuzzy generalized semi-preclosed (in short, Fgsp-closed) [10] if $\operatorname{spCl}(A) \leq$ $H$, whenever $A \leq H$ and $H$ is fuzzy open set in $X$;

7. Fuzzy gegneralized pre closed set (in short, Fgp-closed) [7] if $p C l(A) \leq$ $H$, whenever $A \leq H$ and $H$ is fuzzy open set in $X$;

8. Fuzzy $\omega$-closed(in short, Fw-closed) [19] if $\mathrm{Cl}(A) \leq H$, whenever $A \leq H$ and $H$ is Fs-open set in $X$.

Definition 2.4. A fuzzy topological space $(X, \tau)$ is called a

1. Fuzzy $T_{\frac{1}{2}}$ space [2] if every Fg-closed set in it is fuzzy closed.

2. Fuzzy $T_{\omega}$ space if every F $\omega$-closed set in it is fuzzy closed.

3. Fuzzy $T_{b}$ space if every Fgs-closed set in it is fuzzy closed. 
Definition 2.5. A mapping $f:(X, \tau) \rightarrow(Y, \sigma)$ is said to be:

1. Fg-continuous [2] if $f^{-1}(V)$ is fuzzy closed set in $X$, for every fuzzy closed set $V$ in $Y$;

2. Fsg-continuous [17] if $f^{-1}(V)$ is Fsg-closed in $X$, for each fuzzy closed set $V$ in $Y$;

3. Fgsp-continuous [12] if $f^{-1}(V)$ is Fgsp-closed in $X$, for every fuzzy closed set $V$ in $Y$;

Definition 2.6. [10] A fuzzy point $x_{\lambda} \in A$ is said to be quasi-coincident (in short $q$-coincident) with the fuzzy set $A$ is denoted by $x_{\lambda} q A$ if and only if $\lambda+A(x)>1$. A fuzzy set $A$ is quasi-coincident with a fuzzy set $B$ denoted by $A q B$ if and only if there exists $x \in X$ such that $A(x)+B(x)>1$. If the fuzzy sets $A$ and $B$ are not quasi-coincident then we write $A \bar{q} B$. A fuzzy set $B$ is said to be a $q$-neibourhood (in short, $q-n b d$ ) of a fuzzy set $A$ if there is a fuzzy open sets $U$ with $A q U \leq B$.

Lemma 2.7. [21] Let $A, B, C$ are fuzzy sets in $(X, \tau)$. Then $A q(B \vee C)$ if and only if $A q B$ or $A q C$.

Definition 2.8. [5] Let $f$ be a mapping from $X$ into $Y$. If $A$ is a fuzzy set of $X$ and $B$ is a fuzzy set of $Y$, then

1. $f(A)$ is a fuzzy set of $Y$, where

$$
f(A)= \begin{cases}\sup _{x \in f^{-1}(y)} A(x), & \text { for } f^{-1}(y) \neq 0 \\ 0, & \text { otherwise }\end{cases}
$$

2. $f^{-1}(B)$ is fuzzy set of $X$, where $f^{-1}(B)(x)=B(f(x))$ for each $x \in X$.

3. $f^{-1}(1-B)=1-f^{-1}(B)$.

\section{Fuzzy Generalized sg-Closed sets and Fuzzy Generalized sg-Open Sets}

In this section we introduce and study the fuzzy generalized sg-closed sets, generalized sg-open sets and some of its properties. 
Definition 3.1. A fuzzy set $A$ of $(X, \tau)$ is called a fuzzy generalized sgclosed set (in short, Fgsg-closed) if $\mathrm{Cl}(\mathrm{A}) \leq H$ whenever $A \leq H$ and $H$ is Fsg-open in $X$.

Proposition 3.2. Every fuzzy closed set is Fgsg-closed.

Proof. Let A be fuzzy closed set and $\mathrm{H}$ be any Fsg-open set such that $\mathrm{A} \leq$ $\mathrm{H}$. Since A is fuzzy closed, $\mathrm{Cl}(\mathrm{A})=\mathrm{A} \leq \mathrm{H}$. Hence A is Fgsg-closed.

The reverse implication of the above proposition is not true as shown in the following example.

Example 3.3. Let $X=\{a, b, c\}$ and the fuzzy sets $A, B$ and $C$ from $X$ to $[0,1]$ be defined as $A(a)=0.0, A(b)=0.0, A(c)=0.4 ; B(a)=0.9, B(b)=0.6$, $B(c)=0.0 ; C(a)=1.0, C(b)=0.7, C(c)=1.0$. Let $\tau=\{0, A, B, A \vee B, 1\}$. Then the set $C$ is Fgsg-closed but not fuzzy closed in $(X, \tau)$.

Proposition 3.4. Every Fgsg-closed set is Fg-closed.

Proof. Let A be any Fgsg-closed set and $\mathrm{H}$ be any fuzzy open set such that $\mathrm{A} \leq \mathrm{H}$. Since every fuzzy open set is Fsg-open and A is Fgsg-closed, we have $\mathrm{Cl}(\mathrm{A}) \leq \mathrm{H}$. Hence A is Fg-closed.

Proposition 3.5. Every Fgsg-closed set is Fw-closed.

Proof. Let A be any Fgsg-closed set and $\mathrm{H}$ be any Fs-open set such that $\mathrm{A} \leq \mathrm{H}$. Since every fuzzy semi open set is Fsg-open and A is Fgsg-closed, we have $\mathrm{Cl}(\mathrm{A}) \leq \mathrm{H}$. Hence $\mathrm{A}$ is $\mathrm{F} \omega$-closed.

Proposition 3.6. Every Fgsg-closed set is Fga-closed.

Proof. Let A be any Fgsg-closed set and $\mathrm{H}$ be any fuzzy open set such that $\mathrm{A} \leq \mathrm{H}$. Since every fuzzy open set is Fsg-open and A is Fgsg-closed, we have $\alpha \mathrm{Cl}(\mathrm{A}) \leq \mathrm{Cl}(\mathrm{A}) \leq \mathrm{H}$. Hence $\mathrm{A}$ is $\mathrm{Fg} \alpha$-closed.

Proposition 3.7. Every Fgsg-closed set is Fag-closed.

Proof. Let A be any Fgsg-closed set and $\mathrm{H}$ be any $\mathrm{F} \alpha$-open set such that $\mathrm{A} \leq \mathrm{H}$. Since every $\mathrm{F} \alpha$-open set is fuzzy semi open set which is Fsg-open and $\mathrm{A}$ is Fgsg-closed, we have $\alpha \mathrm{Cl}(\mathrm{A}) \leq \mathrm{Cl}(\mathrm{A}) \leq \mathrm{H}$. Hence $\mathrm{A}$ is $\mathrm{F} \alpha$ g-closed.

Proposition 3.8. Every Fgsg-closed set is Fsg-closed and Fsp-closed. 
Proof. Let A be any Fgsg-closed set and $\mathrm{H}$ be any Fs-open set such that $\mathrm{A} \leq \mathrm{H}$. Since every fuzzy semi open set is Fsg-open and A is Fgsg-closed, we have $\mathrm{sCl}(\mathrm{A}) \leq \mathrm{Cl}(\mathrm{A}) \leq \mathrm{H}$. Hence $\mathrm{A}$ is Fsg-closed. Since every Fsg-closed set is Fsp-closed, A is Fsp-closed.

Proposition 3.9. Every Fgsg-closed set is Fgs-closed,Fgsp-closed and Fgp-closed.

Proof. Let A be any Fgsg-closed set and $\mathrm{H}$ be any fuzzy open set such that $\mathrm{A} \leq \mathrm{H}$. Since every fuzzy open set is Fsg-open and A is Fgsg-closed, we have $\mathrm{sCl}(\mathrm{A}) \leq \mathrm{Cl}(\mathrm{A}) \leq \mathrm{H}$. Hence $\mathrm{A}$ is Fgs-closed

Similarly we have $\operatorname{spCl}(\mathrm{A}) \leq \mathrm{Cl}(\mathrm{A}) \leq \mathrm{H}$ and $\mathrm{pCl}(\mathrm{A}) \leq \mathrm{Cl}(\mathrm{A}) \leq \mathrm{H}$. Hence $\mathrm{A}$ is Fgsp-closed and Fgp-closed .

The following example serves the reverse implications of the above propositions are not true.

Example 3.10. Let $X=\{a, b, c\}$ and the fuzzy sets $A, B, C, D$ and $E$ from $X$ to $[0,1]$ be defined as $A(a)=0.7, A(b)=0.3, A(c)=1.0 ; B(a)=0.7, B(b)=$ $0.0, B(c)=0.0 ; C(a)=0.9, C(b)=0.2, C(c)=0.1 ; D(a)=0.2, D(b)=0.7$, $D(c)=0.0 ; E(a)=0.2, E(b)=0.7, E(c)=0.2$. Let $\tau=\{0, A, B, 1\}$. Then $C$ is Fg-closed and hence Fga-closed, Fgp-closed, Fgs-closed and Fgsp-closed, but not Fgsg-closed in $(X, \tau)$. The fuzzy set $D$ is $F \omega$-closed but not Fgsg-closed in $(X, \tau)$. And the set $E$ is Fag-closed and hence Fsg-closed and Fsp-closed but not Fgsg-closed in $(X, \tau)$.

Definition 3.11. A fuzzy set $A$ of a fuzzy toplogical space $(X, \tau)$ is called Fgsg-open set if and only if $1-A$ is Fgsg-closed.

Proposition 3.12. Every fuzzy open set is Fgsg-open.

Proof. Let A be any fuzzy open set. Let $\mathrm{H}$ be any Fsg-open set such that $1-\mathrm{A} \leq \mathrm{H}$. Since $\mathrm{A}$ is fuzzy open ,we have $\mathrm{Cl}(1-\mathrm{A})=1-\operatorname{Int}(\mathrm{A})=1-\mathrm{A} \leq$ H. This gives $1-\mathrm{A}$ is Fgsg-closed and hence A is Fgsg-open .

Proposition 3.13. Every Fgsg-open set is Fg-open and Fw-open.

Proof. Let A be any Fgsg-open set. Then 1- A is Fgsg-closed. By Propositions 3.4 and 3.5, 1- A is Fg-closed and F $\omega$-closed. Hence A is Fg-open and F $\omega$-open.

Proposition 3.14. Every Fgsg-open set is Fgs-open, Fsg-open, Fsp-open, Fgsp-open, Fga-open and Fag-open. 
Proof. Similar to above Proposition.

\section{Characterization of Fgsg-Closed Sets and Fgsg-Open Sets}

In this section we study several interesting characterizations of fuzzy generalized sg-closed sets and fuzzy generalized sg-open sets.

Definition 4.1. A fuzzy set $A$ in $(X, \tau)$ is called Fgsg-nhd of a fuzzy point $x_{\lambda}$ if there exixts a Fgsg-open set $B$ such that $x_{\lambda} \in B \leq A$. A Fgsg-nhd $A$ is said to be Fgsg-open-nhd (resp.Fgsg-closed-nhd) if and only if A is Fgsgopen(resp.Fgsg-closed). A fuzzy set $A$ in $(X, \tau)$ is called fuzzy gsg-q-nhd of a fuzzy point $x_{\lambda}$ (resp.fuzzy set $B$ ), if there exists a Fgsg-open set $U$ in $(X, \tau)$ such that $x_{\lambda} q U \leq A$ (resp. $\left.B q U \leq A\right)$.

Theorem 4.2. If $A$ and $B$ are Fgsg-closed sets in $(X, \tau)$ then $A \vee B$ is Fgsg-closed.

Proof. Let A and B be two fuzzy Fgsg-closed sets in (X, $\tau)$ and let $\mathrm{H}$ be any Fsg-open set such that $\mathrm{A} \leq \mathrm{H}$ and $\mathrm{B} \leq \mathrm{H}$. Therefore we have $\mathrm{Cl}(\mathrm{A}) \leq \mathrm{H}$ and $\mathrm{Cl}(\mathrm{B}) \leq \mathrm{H}$. Since $\mathrm{A} \leq \mathrm{H}$ and $\mathrm{B} \leq \mathrm{H}$, wehave $\mathrm{A} \vee \mathrm{B} \leq \mathrm{H}$. Now $\mathrm{Cl}(\mathrm{A} \vee \mathrm{B})=$ $\mathrm{Cl}(\mathrm{A}) \vee \mathrm{Cl}(\mathrm{B}) \leq \mathrm{H}$. Hence $\mathrm{A} \vee \mathrm{B}$ is Fgsg-closed.

Theorem 4.3. If $A$ and $B$ are Fgsg-open sets in $(X, \tau)$ then $A \wedge B$ is Fgsg-open.

Proof. Let A and B be two fuzzy Fgsg-open sets in $(\mathrm{X}, \tau)$. Then $1-\mathrm{A}$ and $1-\mathrm{B}$ are Fgsg-closed. By above Theorem 4.2, $(1-\mathrm{A}) \vee(1-\mathrm{B})$ is Fgsg-closed. Since $(1-\mathrm{A}) \vee(1-\mathrm{B})=1-(\mathrm{A} \wedge \mathrm{B})$. Hence $\mathrm{A} \wedge \mathrm{B}$ is Fgsg-open.

Theorem 4.4. If a fuzzy set $A$ is Fgsg-closed in $(X, \tau)$ and $C l(A) \wedge(1$ $-C l(A))=0$ then $C l(A)-A$ does not contain any non-zero Fsg-closed set in $(X, \tau)$.

Proof. Let A be Fgsg-closed in $(\mathrm{X}, \tau)$ and $\mathrm{Cl}(\mathrm{A}) \wedge(1-\mathrm{Cl}(\mathrm{A}))=0$. We prove the result by contradiction. Let $\mathrm{B}$ be any Fsg-closed set in $(\mathrm{X}, \tau)$ such that $\mathrm{B} \leq \mathrm{Cl}(\mathrm{A})-\mathrm{A}$ and $\mathrm{B} \neq 0$. This gives $\mathrm{B} \leq \mathrm{Cl}(\mathrm{A})$ and $\mathrm{B} \leq 1-\mathrm{A}$. We have $\mathrm{A} \leq 1-\mathrm{B}$, which is Fsg-open. Since A is Fgsg-closed, we have $\mathrm{Cl}(\mathrm{A}) \leq 1-\mathrm{B}$. This implies $\mathrm{B} \leq 1-\mathrm{Cl}(\mathrm{A})$. Therefore $\mathrm{B} \leq \mathrm{Cl}(\mathrm{A}) \wedge 1-\mathrm{Cl}(\mathrm{A})=0$. That is $\mathrm{B}$ $=0$, which is a contradiction. Hence $\mathrm{Cl}(\mathrm{A})-\mathrm{A}$ does not contain any non-zero Fsg-closed set in $(\mathrm{X}, \tau)$. 
Theorem 4.5. If a fuzzy set $A$ is Fgsg-closed in $(X, \tau)$ and $C l(A) \wedge$ $(1-C l(A))=0$ then $C l(A)-A$ does not contain any non-zero fuzzy closed set in $(X, \tau)$.

Proof. It follows from the above theorem and the fact that every fuzzy closed set is Fsg-closed.

Theorem 4.6. If $A$ is Fsg-open and Fgsg-closed in $(X, \tau)$ then $A$ is fuzzy closed in $(X, \tau)$.

Proof. Since $\mathrm{A} \leq \mathrm{A}$ and $\mathrm{A}$ is Fsg-open and Fgsg-closed, we have $\mathrm{Cl}(\mathrm{A}) \leq$ A. Since $\mathrm{A} \leq \mathrm{Cl}(\mathrm{A})$, we have $\mathrm{A}=\mathrm{Cl}(\mathrm{A})$. Hence $\mathrm{A}$ is fuzzy closed.

Theorem 4.7. A fuzzy set $A$ of $(X, \tau)$ is Fgsg-closed if and only if $A \bar{q} U$ $\Rightarrow C l(A) \bar{q} U$, for every $F_{s g}$-closed set $U$ of $(X, \tau)$.

Proof. (Necessity) Let U be Fsg-closed set and $A \bar{q} U$. Then $\mathrm{A} \leq 1-\mathrm{U}$. Since $\mathrm{A}$ is Fgsg-closed and $1-\mathrm{U}$ is Fsg-open, we have $\mathrm{Cl}(\mathrm{A}) \leq 1-\mathrm{U}$. Hence $\mathrm{Cl}(A) \bar{q} U$. (Sufficiency) Let $\mathrm{H}$ be Fsg-open set such that $\mathrm{A} \leq \mathrm{H}$. By hypothesis $A \bar{q}(1-H)$ $\Rightarrow \mathrm{Cl}(A) \bar{q}(1-H)$, as $1-\mathrm{H}$ is Fsg-closed. Then $\mathrm{Cl}(\mathrm{A}) \leq \mathrm{H}$. Hence $\mathrm{A}$ is Fgsgclosed.

Theorem 4.8. If $A$ is Fgsg-closed set in $(X, \tau)$ and $A \leq B \leq C l(A)$ then $B$ is Fgsg-closed in $(X, \tau)$.

Proof. Let $\mathrm{H}$ be Fsg-open set such that $\mathrm{B} \leq \mathrm{H}$. Since $\mathrm{A} \leq \mathrm{B}$, we have $\mathrm{A}$ $\leq \mathrm{H}$. Since $\mathrm{A}$ is Fgsg-closed set, $\mathrm{Cl}(\mathrm{A}) \leq \mathrm{H}$. But $\mathrm{B} \leq \mathrm{Cl}(\mathrm{A})$ implies $\mathrm{Cl}(\mathrm{B}) \leq$ $\mathrm{Cl}(\mathrm{Cl}(\mathrm{A}))=\mathrm{Cl}(\mathrm{A}) \leq \mathrm{H}$. Hence $\mathrm{B}$ is Fgsg-closed.

Theorem 4.9. If $A$ is Fgsg-open set in $(X, \tau)$ and $\operatorname{Int}(A) \leq B \leq A$, then $B$ is Fgsg-open in $(X, \tau)$.

Proof. Let $\mathrm{A}$ is Fgsg-open set in $(\mathrm{X}, \tau)$ and $\operatorname{Int}(\mathrm{A}) \leq \mathrm{B} \leq \mathrm{A}$. Then $1-\mathrm{A}$ is Fgsg-closed and $1-A \leq 1-B \leq \mathrm{Cl}(1-\mathrm{A})$. Then by theorem $4.8,1-\mathrm{B}$ is Fgsg-closed. Hence B is Fgsg-open.

Theorem 4.10. A fuzzy set $A$ is Fgsg-open if and only if $F \leq \operatorname{Int}(A)$ where $F$ is Fgsg-closed and $F \leq A$. 
Proof. Let $\mathrm{F} \leq \operatorname{Int}(\mathrm{A})$ where $\mathrm{F}$ is Fgsg-closed and $\mathrm{F} \leq \mathrm{A}$. Then $1-A \leq 1-F$ and $1-F$ is Fsg-open. Now $\mathrm{Cl}(1-A)=1-\operatorname{Int}(\mathrm{A}) \leq 1-F$, by hypothesis. Then $1-A$ is Fgsg-closed. Hence $\mathrm{A}$ is Fgsg-open.

Conversely, let $\mathrm{A}$ is Fgsg-open and $\mathrm{F}$ is Fsg-closed and $\mathrm{F} \leq \mathrm{A}$. Then $1-A \leq$ $1-F$. Since $1-A$ is Fgsg-closed and $1-F$ is Fsg-open, we have $\mathrm{Cl}(1-A) \leq 1-F$. Then $\mathrm{F} \leq \operatorname{Int}(\mathrm{A})$.

Theorem 4.11. If $A$ be a Fgsg-closed set in $(X, \tau)$ and $x_{\lambda}$ be a fuzzy point of $X$ such that $x_{\lambda} q \mathrm{Cl}(\mathrm{A})$ then $\mathrm{Cl}\left(x_{\lambda}\right) q A$.

Proof. Suppose $\mathrm{Cl}\left(x_{\lambda}\right) \bar{q} \mathrm{~A}$ then $\mathrm{A} \leq 1-\mathrm{Cl}\left(x_{\lambda}\right)$. Since $1-\mathrm{Cl}\left(x_{\lambda}\right)$ is Fsg-open and $\mathrm{A}$ is Fgsg-closed, we have $\mathrm{Cl}(\mathrm{A}) \leq 1-\mathrm{Cl}\left(x_{\lambda}\right)=1-x_{\lambda}$. This gives $x_{\lambda} \bar{q} \mathrm{Cl}(\mathrm{A})$, a contradiction. Hence $\mathrm{Cl}\left(x_{\lambda}\right) \mathrm{qA}$.

\section{Fgsg-Closure and Fgsg-Interior}

We introduce fuzzy gsg-closure and fuzzy gsg-interior and obtain some of its properties in this section.

Fuzzy gsg-closure and fuzzy gsg-interior of fuzzy set A in fuzzy topological space $(\mathrm{X}, \tau)$ is denoted by gsg- $\mathrm{Cl}(\mathrm{A})$ and gsg-Int $(\mathrm{A})$ respectively and defined as follows:

Definition 5.1. Let $A$ be any fuzzy set in $(X, \tau)$ then we define FgsgClosure and Fgsg-Interior as

$\operatorname{gsg}-C l(A)=\wedge\{B: B$ is Fgsg-closed and $B \geq A\}$, $\operatorname{gsg}-\operatorname{Int}(A)=\vee\{B: B$ is Fgsg-open and $B \leq A\}$.

It is evident that

1. $\operatorname{gsg}-\mathrm{Cl}(\mathrm{A})=\mathrm{A}$ if and only if A is Fgsg-closed.

2. $\operatorname{gsg}-\operatorname{Int}(\mathrm{A})=\mathrm{A}$ if and only if $\mathrm{A}$ is Fgsg-open.

3. gsg- $\mathrm{Cl}(\mathrm{A})$ is the smallest fuzzy set containing A.

4. gsg-Int(A) is the largest fuzzy set contained in A.

Theorem 5.2. Let $x_{\lambda}$ and $A$ be a fuzzy point and fuzzy set respectively in $(X, \tau)$. Then $x_{\lambda} \in g s g-C l(A)$ if and only if every fuzzy gsg-q-nhd of $x_{\lambda}$ is q-coincident with $A$. 
Proof. We prove by contradiction. Let $x_{\lambda} \leq$ gsg-Cl(A). Suppose there exists a gsg-q-nhd $\mathrm{U}$ of $x_{\lambda}$ such that $\mathrm{U} \bar{q} \mathrm{~A}$. Since $\mathrm{U}$ is gsg-q-nhd of $x_{\lambda}$, there exists Fgsg-open set $\mathrm{V}$ in $(\mathrm{X}, \tau)$ such that $x_{\lambda} \mathrm{qV} \leq \mathrm{U}$ whish gives that $\mathrm{V} \bar{q} \mathrm{~A}$ and hence $\mathrm{A} \leq 1-\mathrm{V}$. Then gsg- $\mathrm{Cl}(\mathrm{A}) \leq 1-\mathrm{V}$, as $1-\mathrm{V}$ is Fgsg-closed. Since $x_{\lambda} \notin 1-\mathrm{V}$ , we have $x_{\lambda} \notin$ gsg-Cl(A), a contradiction. Hence every fuzzy gsg-q-nhd of $x_{\lambda}$ is q-coincident with $\mathrm{A}$.

Conversely suppose $x_{\lambda} \notin$ gsg-Cl(A). Then There exists a Fgsg-closed set B such that $\mathrm{A} \leq \mathrm{B}$ and $x_{\lambda} \notin \mathrm{B}$.Then we have $x_{\lambda} \mathrm{q}(1-\mathrm{B})$ and $\mathrm{A} \bar{q}(1-\mathrm{B})$, a contradiction. Hence $x_{\lambda} \in$ gsg- $\mathrm{Cl}(\mathrm{A})$.

Properties 5.3. Let $A$ be any fuzzy set in $(X, \tau)$. Then $\operatorname{gsg}-\operatorname{Int}(1-A)=1-(\operatorname{gsg}-C l(A))$. $\operatorname{gsg}-\mathrm{Cl}(1-A)=1-(\operatorname{gsg}-\operatorname{Int}(A))$.

Proof. (i)By definition, gsg- $\mathrm{Cl}(\mathrm{A})=\wedge\{\mathrm{B}: \mathrm{B}$ is Fgsg-closed and $B \geq A\}$

$$
\begin{aligned}
1-\operatorname{gsg}-\mathrm{Cl}(\mathrm{A}) & =1-\wedge\{\mathrm{B}: \mathrm{B} \text { is Fgsg-closed and } \mathrm{B} \geq \mathrm{A}\} \\
& =\vee\{1-\mathrm{B}: \mathrm{B} \text { is Fgsg-closed and } \mathrm{B} \geq A\} \\
& =\vee\{\mathrm{U}: \mathrm{U} \text { is Fgsg-open and } \mathrm{U} \leq 1-\mathrm{A}\} \\
& =\operatorname{gsg}-\operatorname{Int}(1-\mathrm{A})
\end{aligned}
$$

(ii) The proof is similar to (i)

Properties 5.4. If $A$ and $B$ are Fuzzy sets in $(X, \tau)$. Then the following are true.

1. $g s g-C l(0)=0, g s g-C l(1)=1$.

2. $g s g-C l(A)$ is $F_{g s g}$-closed in $(X, \tau)$.

3. $g s g-C l(A) \leq g s g-C l(B)$ when $A \leq B$.

4. $U q A$ if and only if $U q g s g-C l(A)$, when $U$ is $F_{g s g}$-open set in $(X, \tau)$.

5. $g s g-C l(A)=g s g-C l(g s g-C l(A))$.

6. $g s g-C l(A \wedge B) \leq g s g-C l(A) \wedge g s g-C l(B)$.

7. $g s g-C l(A \vee B)=g s g-C l(A) \vee g s g-C l(B)$.

Proof. 1. and

2. are obvious. 
3. Let $x_{\lambda} \notin$ gsg-Cl(B).Then by above theorem 5.2 , there exist fuzzy gsgq-nhd $\mathrm{V}$ of $x_{\lambda}$ such that $\mathrm{V} \bar{q} \mathrm{~B}$. Since $\mathrm{V}$ is fuzzy gsg-q-nhd $\mathrm{V}$ of $x_{\lambda}$ there exists fuzzy open set $\mathrm{U}$ such that $x_{\lambda} \mathrm{q} \mathrm{U} \leq \mathrm{V}$. This gives $\mathrm{U} \bar{q} \mathrm{~B}$. Since $\mathrm{A} \leq \mathrm{B}$, then $\mathrm{U} \bar{q} \mathrm{~A}$. Then by above theorem $5.2, x_{\lambda} \notin$ gsg-Cl(A).Hence gsg-Cl(A) $\leq$ $\operatorname{gsg}-\mathrm{Cl}(\mathrm{B})$.

4. Let $\mathrm{U}$ be any Fgsg-open set in $(\mathrm{X}, \tau)$.Suppsoe $\mathrm{U} \bar{q} \mathrm{~A}$, then $\mathrm{A} \leq 1-\mathrm{U}$.Since $1-\mathrm{U}$ is Fgsg-closed and by (iii), gsg- $\mathrm{Cl}(\mathrm{A}) \leq \operatorname{gsg}-\mathrm{Cl}(1-U)=1-\mathrm{U}$. Thus $\mathrm{U} \bar{q}$ gsg-Cl(A). Conversely, Let $\mathrm{U} \bar{q}$ gsg- $\mathrm{Cl}(\mathrm{A})$. Then gsg- $\mathrm{Cl}(\mathrm{A}) \leq 1-\mathrm{U}$. Since $\mathrm{A} \leq \mathrm{gsg}-\mathrm{Cl}(\mathrm{A})$, we have $\mathrm{A} \leq 1-\mathrm{U}$. Thus $\mathrm{U} \bar{q} \mathrm{~A}$. Hence $\mathrm{UqA}$ if and only if $\mathrm{Uq}$ gsg- $\mathrm{Cl}(\mathrm{A})$.

5. Since gsg- $\mathrm{Cl}(\mathrm{A}) \leq \mathrm{gsg}-\mathrm{Cl}(\mathrm{gsg}-\mathrm{Cl}(\mathrm{A}))$, it is enough to prove gsg- $\mathrm{Cl}$ (gsg$\mathrm{Cl}(\mathrm{A})) \leq \operatorname{gsg}-\mathrm{Cl}(\mathrm{A})$. Let $x_{\lambda} \notin \operatorname{gsg}-\mathrm{Cl}(\mathrm{A})$. Then by theorem 5.2, there exist fuzzy gsg-q-nhd $\mathrm{V}$ of $x_{\lambda}$ such that $\mathrm{V} \bar{q} \mathrm{~A}$ and so there is a Fgsg-open set $\mathrm{U}$ in $(\mathrm{X}, \tau)$ such that $x_{\lambda} \mathrm{qU} \leq \mathrm{V}$ and $\mathrm{U} \bar{q} \mathrm{~A}$. By (iv), $\mathrm{U} \bar{q}$ gsg-Cl(A). Then by theorem $5.2 x_{\lambda} \notin$ gsg-Cl(gsg-Cl(A)). Hence gsg-Cl(A) = gsg-Cl(gsg$\mathrm{Cl}(\mathrm{A}))$.

6. Since $\mathrm{A} \wedge \mathrm{B} \leq \mathrm{A}$ and $\mathrm{A} \wedge \mathrm{B} \leq \mathrm{B}$, gsg- $\mathrm{Cl}(\mathrm{A} \wedge \mathrm{B}) \leq \operatorname{gsg}-\mathrm{Cl}(\mathrm{A})$ and gsg$\mathrm{Cl}(\mathrm{A} \wedge \mathrm{B}) \leq \operatorname{gsg}-\mathrm{Cl}(\mathrm{B})$. Hence gsg- $\mathrm{Cl}(\mathrm{A} \wedge \mathrm{B}) \leq \operatorname{gsg}-\mathrm{Cl}(\mathrm{A}) \wedge$ gsg-Cl$(\mathrm{B})$.

7. Since $\mathrm{A} \leq \mathrm{A} \vee \mathrm{B}$ and $\mathrm{B} \leq \mathrm{A} \vee \mathrm{B}$, gsg- $\mathrm{Cl}(\mathrm{A}) \leq \operatorname{gsg}-\mathrm{Cl}(\mathrm{A} \vee \mathrm{B})$ and gsg- $\mathrm{Cl}(\mathrm{B})$

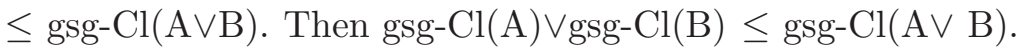

Conversely, $x_{\lambda} \in \operatorname{gsg}-\mathrm{Cl}(\mathrm{A} \vee \mathrm{B})$. Then by theorem 5.2, there exist fuzzy gsg-q-nhd $\mathrm{U}$ of $x_{\lambda}$ such that $\mathrm{Uq}(\mathrm{A} \vee \mathrm{B})$. By Lemma 2.7, either UqA or UqB. Then by theorem $5.2, x_{\lambda} \in$ gsg-Cl(A) or $x_{\lambda} \in$ gsg-Cl(B). That is $x_{\lambda} \in$ gsg-Cl(A) $\vee$ gsg-Cl(B).Then gsg- $\mathrm{Cl}(\mathrm{A} \vee \mathrm{B}) \leq \mathrm{gsg}-\mathrm{Cl}(\mathrm{A}) \vee \mathrm{gsg}-\mathrm{Cl}(\mathrm{B})$. Hence $\operatorname{gsg}-\mathrm{Cl}(\mathrm{A} \vee \mathrm{B})=\operatorname{gsg}-\mathrm{Cl}(\mathrm{A}) \vee \operatorname{gsg}-\mathrm{Cl}(\mathrm{B})$.

Properties 5.5. If $A$ and $B$ are Fuzzy sets in $(X, \tau)$. Then the following are true.

1. $\operatorname{gsg}-\operatorname{Int}(0)=0, \operatorname{gsg}-\operatorname{Int}(1)=1$.

2. $\operatorname{gsg}$-Int(A) is Fgsg-open in $(X, \tau)$.

3. $\operatorname{gsg}-\operatorname{Int}(A) \leq \operatorname{gsg}-\operatorname{Int}(B)$ when $A \leq B$.

4. $\operatorname{gsg}-\operatorname{Int}(A)=g \operatorname{sg}-\operatorname{Int}(\operatorname{gsg}-\operatorname{Int}(A))$.

5. $\operatorname{gsg}-\operatorname{Int}(A \vee B) \geq g \operatorname{sg}-\operatorname{Int}(A) \vee \operatorname{gsg}-\operatorname{Int}(B)$. 
6. $\operatorname{gsg}-\operatorname{Int}(A \wedge B)=\operatorname{gsg}-\operatorname{Int}(A) \wedge \operatorname{gsg}-\operatorname{Int}(B)$.

Proof. Obvious.

\section{Fuzzy Tgsg Space}

In this section we introduce fuzzy Tgsg-space as an application of fuzzy generalized sg-closed set.

Definition 6.1. A fuzzy topological space $(X, \tau)$ is called a Fuzzy $T_{g s g}$ Space if every Fgsg-closet set in it is fuzzy closed.

Proposition 6.2. Every fuzzy $T_{\frac{1}{2}}$ space is fuzzy Tgsg space.

Proof. Let $(\mathrm{X}, \tau)$ be a fuzzy $T_{\frac{1}{2}}$ space and let A be Fgsg-closed set in $(\mathrm{X}, \tau)$. Then A is Fg-closed,by proposition 3.4. Since $(\mathrm{X}, \tau)$ is $T_{\frac{1}{2}}$ space, A is fuzzy closed in $(\mathrm{X}, \tau)$. Hence $(\mathrm{X}, \tau)$ is fuzzy $T_{g s g}$ space.

Proposition 6.3. Every fuzzy $T_{\omega}$ space is a fuzzy Tgsg space.

Proof. Let $(\mathrm{X}, \tau)$ be a fuzzy $T_{\omega}$ space and let A be Fgsg-closed set in $(\mathrm{X}, \tau)$.Then $\mathrm{A}$ is $\mathrm{F} \omega$-closed,by proposition 3.5. Since $(\mathrm{X}, \tau)$ is $T_{\omega}$ space, $\mathrm{A}$ is fuzzy closed in $(\mathrm{X}, \tau)$. Hence $(\mathrm{X}, \tau)$ is fuzzy $T_{g s g}$ space.

Proposition 6.4. Every fuzzy $T_{b}$ space is a fuzzy Tgsg space.

Proof. Let $(\mathrm{X}, \tau)$ be a fuzzy $T_{b}$ space and let A be Fgsg-closed set in $(\mathrm{X}, \tau)$.Then $\mathrm{A}$ is Fgs-closed,by proposition 3.9. Since $(\mathrm{X}, \tau)$ is $T_{b}$ space, $\mathrm{A}$ is fuzzy closed in $(\mathrm{X}, \tau)$. Hence $(\mathrm{X}, \tau)$ is fuzzy $T_{g s g}$ space.

The following example shows that the converse of the above theorems is not true.

Example 6.5. Let $X=\{a, b, c\}$ and the fuzzy sets $A$ and $B$ from $X$ to $[0,1]$ be defined as $A(\mathrm{a})=0.7, A(b)=0.3, A(c)=1.0 ; B(a)=0.7, B(b)=0.0$, $B(c)=0.0 ;$ Let $\tau=\{0, A, B, 1\}$. Then $(X, \tau)$ is $T_{g s g}$ but not $T_{\frac{1}{2}}$ space, $T_{\omega}$ space and $T_{b}$ space. 


\section{Fgsg-Continuous and Fgsg-Irresolute Mappings}

We introduce Fgsg-continuous and Fgsg-irresolute mappings and study some of their fundamental properties in this section.

Definition 7.1. A mapping $f:(X, \tau) \rightarrow(Y, \sigma)$ is called fuzzy gsgcontinuous (in short, Fgsg-continuous) if $f^{-1}(V)$ is Fgsg-closed in $(X, \tau)$ for every fuzzy closed set $V$ of $(Y, \sigma)$.

Definition 7.2. A mapping $f:(X, \tau) \rightarrow(Y, \sigma)$ is called fuzzy gsgirresolute(in short, Fgsg-irresolute) if $f^{-1}(V)$ is Fgsg-closed in $(X, \tau)$ for every Fgsg-closed set $V$ of $(Y, \sigma)$.

Theorem 7.3. Let $f:(X, \tau) \rightarrow(Y, \sigma)$ be Fgsg-continuous. Then $f$ is Fsg-continuous.

Proof. Let $\mathrm{V}$ be a fuzzy closed set in $(Y, \sigma)$. Since $f$ is Fgsg-continuous, $f^{-1}(V)$ is Fgsg-closed in $(X, \tau)$. By proposition 3.8, $f^{-1}(V)$ is Fsg-closed in $(X, \tau)$. Hence $f$ is Fsg-continuous.

The converse of the above theorem is not true in general. For,

Example 7.4. Let $X=\{a, b\}, Y=\{x, y\}$. Fuzzy sets $A$ is defined as $A(a)=0.4, A(b)=0.6$. Let $\tau=\{0, A, 1\}$ and $\sigma=\{0,1\}$. Then the mapping $f:(X, \tau) \rightarrow(Y, \sigma)$ defined by $f(a)=x, f(b)=y$ is Fgsg-continuous but not Fgsg-irresolute.

Theorem 7.5. Letf $:(X, \tau) \rightarrow(Y, \sigma)$ be Fgsg-continuous. Then $f$ is Fgsp-continuous

Proof. Let $\mathrm{V}$ be a fuzzy closed set in $(Y, \sigma)$. Since $f$ is Fgsg-continuous, $f^{-1}(V)$ is Fgsg-closed in $(X, \tau)$. By proposition 3.9, $f^{-1}(V)$ is Fgsp-closed in $(X, \tau)$. Hence $f$ is Fgsp-continuous.

The converse of the above theorem is not true in general. For,

Example 7.6. Let $X=\{a, b\}, Y=\{x, y\}$. Fuzzy sets $A$ and $B$ are defined as $A(a)=0.3, A(b)=0.7 ; B(x)=0.3, B(y)=0.4$. Let $\tau=\{0, A, 1\}$ and $\sigma=$ $\{0, B, 1\}$. Then the mapping $f:(X, \tau) \rightarrow(Y, \sigma)$ defined by $f(a)=x, f(b)=y$ is Fgsp-continuous but not Fgsg-continuous.

Theorem 7.7. Let $f:(X, \tau) \rightarrow(Y, \sigma)$ be Fgsg-continuous if and only if inverse image of each fuzzy open set of $(Y, \sigma)$ is Fgsg-open in $(X, \tau)$. 
Proof. Let $f$ be Fgsg-continuous. If $\mathrm{V}$ is any fuzzy open set in $(\mathrm{Y}, \sigma)$ then $f^{-1}(1-V)=1-f^{-1}(V)$ is Fgsg-closed. Hence $f^{-1}(V)$ is Fgsg-open in $(X, \tau)$. Conversely, Let $\mathrm{V}$ be a fuzzy closed set in $(Y, \sigma)$. By hypothesis, $f^{-1}(1-V)$ is Fgsg-open in $(X, \tau)$. This gives $f^{-1}(V)$ is Fgsg-closed. Hence $f$ is Fgsgcontinuous.

Theorem 7.8. If $f:(X, \tau) \rightarrow(Y, \sigma)$ is $F_{g s g}$-continuous then for each fuzzy point $x_{\lambda}$ of $X$ and $A \in \sigma$ such that $f\left(x_{\lambda}\right) \in A$, there exists a $F_{g s g}$-open set $B$ of $X$ such that $x_{\lambda} \in B$ and $f(B) \leq A$.

Proof. Let $x_{\lambda}$ be a fuzzy point of $\mathrm{X}$ and $\mathrm{A} \in \sigma$ such that $f\left(x_{\lambda}\right) \in A$. Take $\mathrm{B}=f^{-1}(V)$. Since $1-\mathrm{A}$ is fuzzy closed in $(Y, \sigma)$ and $f$ is Fgsg-continuous, we have $f^{-1}(1-A)=1-f^{-1}(A)$ is Fgsg-closed in $(X, \tau)$. This gives $\mathrm{B}=f^{-1}(A)$ is Fgsg-open in $(X, \tau)$ and $x_{\lambda} \in B$ and $f(B)=f\left(f^{-1}(A)\right) \leq A$.

Theorem 7.9. If $f:(X, \tau) \rightarrow(Y, \sigma)$ is Fgsg-continuous then for each fuzzy point $x_{\lambda}$ of $X$ and $A \in \sigma$ such that $f\left(x_{\lambda}\right) q A$, there exists a Fgsg-open set $B$ of $X$ such that $x_{\lambda} q B$ and $f(B) \leq A$.

Proof. Let $x_{\lambda}$ be a fuzzy point of $\mathrm{X}$ and $A \in \sigma$ such that $f\left(x_{\lambda}\right) q A$. Take $B=f^{-1}(A)$. By above thereon 7.10, B is Fgsg-open in $(X, \tau)$ and $x_{\lambda} q B$ and $f(B)=f\left(f^{-1}(A)\right) \leq \mathrm{A}$.

Theorem 7.10. If $f:(X, \tau) \rightarrow(Y, \sigma)$ is $F_{g s g^{-}}$continuous and $g:(Y, \sigma) \rightarrow$ $(Z, \eta)$ is $F_{g}$-continuous and $(Y, \sigma)$ is a fuzzy $T_{\frac{1}{2}}$ space. Then gof $:(X, \tau) \rightarrow$ $(Z, \eta)$ is $F_{g s g}$-continuous.

Proof. Let $\mathrm{V}$ be a fuzzy closed set in $(Z, \eta)$. Since $g$ is Fg-continuous and $(Y, \sigma)$ is a fuzzy $T_{\frac{1}{2}}$ space, $g^{-1}(V)$ is fuzzy closed in $(Y, \sigma)$. Since $f$ is Fgsg- continuous, $f^{-1}\left(g^{-1}(V)\right)$ is Fgsg-closed in $(X, \tau)$. Hence gof is Fgsgcontinuous.

\section{References}

[1] K. K. Azad, On fuzzy semicontinuity, fuzzy almost continuity and fuzzy weaklycontinuity, J. Math. Anal. Appl., 82, No.1 (1981), 14-32.

[2] G. Balasubramanian and P. Sundaram, On some generalizations of fuzzy continuous functions, Fuzzy sets and systems, 86, No.1 (1997), 93-100. 
[3] S.S.Benchalli, R.S.Wali and Basavaraj M.Ittanagi, On Fuzzy r $\omega$-closed sets and fuzzy rw-open sets in fuzzy topological spaces, Int.J.Math.Sci.Appl., 1, No.2 (2011),1007-1022.

[4] S. Bin Shahna, On fuzzy strong semiocontinuity and fuzzy precontinuity, Fuzzy sets and systems, 44, No.2,(1991), 303-308.

[5] L. Chang, Fuzzy topological spaces,J. Math. Anal. Appl., 24,(1968), 182190.

[6] K. M. Abd EI-Hakeim, Generalized semi-continuous mappings in fuzzy topological spaces, J. Fuzzy Math., 7, No.3 (1999), 577-589.

[7] T.Fukutake, R.K.Saraf, M.Caldas and S.Mishra, Mapppings via Fgp-closed sets, Bull.of kuoda Univ.of Edu., 52, No.3 (2003), 11-20.

[8] M.Lellis Thivagar, Nirmala Rebecca Paul and Saeid Jafari, On New Class of Generalized Closed Sets, Annais of the University of Craiova, Mathematics and Computer Science Series,38, No.3 (2011), 84-93.

[9] H.Maki, T. Fukutake, M. Kojima and H. Harada, Generalized closed sets fuzzy topological spaces I, Meetings on Toplogical Sapces Thoery and its Applications , (1998), 23-36.

[10] S.R. Malghan and S.S.Benchalli, Open Maps, Closed Maps and Local Compactnes in Fuzzy Topological Spaces,Int.J.Math.Anal.Appl. , 99, No.2(1984),74-79.

[11] S.Murugasen and P.Thangavelu, Fuzzy Pre-semi-closed Sets,Bull.Malays.Math.Sci. Sec(2), 31, No.2 (2008), 223-232.

[12] O.Bedre Ozbakir, On Generalized Fuzzy Strongly Semi Closed Sets in Fuzzy Topological Spaces, Int.j.Math.Sci., 30, No.11 (2002), 651-657.

[13] P. M. Pu, and Y. M. lin, Fuzzy topology I. neighborhood structure of a fuzzy point and Moore-Smith convergence,J. Math. Anal. Appl., 76, No.2 (1980), 571-599.

[14] R.K. Saraf, and S. Mishra, Fg $\alpha$-closed sets, J. Tripura Math. Soc., 2, (2000) 27-32.

[15] R.K. Saraf, and M. Khanna, Fuzzy generalized semipreclosed sets, J. Tripura Math. Soc.,3,(2001) 59-68. 
[16] R.K. Saraf, and M. Khanna, On gs-closed sets in fuzzy topology, J. Indian Acad.Math.,25, No.1 (2003), 133-143.

[17] R.K. Saraf and M.Caldas, Preserving Fuzzy Sg-closed sets, Universidad Catolica del Norte Antofagasta-Chile, 25 No.2, (2001),127-138.

[18] R.K. Saraf ,G.Navalagia and M.Khanna, On Fuzzy Semi Pre Generalized Closed Sets, Bull.Malays.Math.Sci.,Sec (2), 28 No.1, (2005) 19-30.

[19] M.Sudha, E.Roja and M.K.Uma, Slightly Fuzzy $\omega$-continuous Mappings, Int. J.of Math. Anal,,5 No.16 (2011), 779 - 787.

[20] Luay A.AL-Swidi and Amed S.A.Oan, Fuzzy $\gamma$ - open sets and Fuzzy $\gamma$ closed sets, American Journal of Scientific Research , 27 (2011),62-67.

[21] S. S. Thakur and S. Singh, On fuzzy semi-preopen sets and fuzzy semiprecontinuity,Fuzzy Sets and systems, 98 No.3 (1998), 383-391.

[22] L. A. Zadeh, Fuzzy sets, Information and control, 8 (1965), 338-353. 